\title{
Metabolomics of Neural Progenitor Cells: A Novel Approach to Biomarker Discovery
}

\author{
M. Maletić-Savatić, ${ }^{* \dagger}$ L.K. Vingara ${ }^{\ddagger}$ L.N. Manganas, ${ }^{*}$ Y. Li, $*$ S. Zhang, $*$ \\ A. Sierra, ${ }^{*}$ R. HAZEL, ${ }^{*}$ D. Smith ${ }^{\S}$ M.E. Wagshul, ${ }^{*}$ F. Henn ${ }^{\S}$ L. Krupp, ${ }^{*}$ \\ G. EniKolopov, ${ }^{\dagger}$ H. Benveniste, ${ }^{\S}$ P.M. DJurić, ${ }^{*}$ And I. PelCZeR ${ }^{\dagger}$ \\ *Departments of Neurology and Electrical and Computer Engineering, Stony Brook University, \\ Stony Brook, New York 11790; ' Cold Spring Harbor Laboratory, Cold Spring Harbor, New York 11724; \\ ${ }^{7}$ Department of Chemistry, Princeton University, Princeton, New Jersey 08544; ${ }^{\$}$ Medical Department, \\ Brookhaven National Laboratory, Upton, New York 11973
}

\begin{abstract}
Finding biomarkers of human neurological diseases is one of the most pressing goals of modern medicine. Most neurological disorders are recognized too late because of the lack of biomarkers that can identify early pathological processes in the living brain. Late diagnosis leads to late therapy and poor prognosis. Therefore, during the past decade, a major endeavor of clinical investigations in neurology has been the search for diagnostic and prognostic biomarkers of brain disease. Recently, a new field of metabolomics has emerged, aiming to investigate metabolites within the cell/tissue/ organism as possible biomarkers. Similarly to other "omics" fields, metabolomics offers substantial information about the status of the organism at a given time point. However, metabolomics also provides functional insight into the biochemical status of a tissue, which results from the environmental effects on its genome background. Recently, we have adopted metabolomics techniques to develop an approach that combines both in vitro analysis of cellular samples and in vivo analysis of the mammalian brain. Using proton magnetic resonance spectroscopy, we have discovered a metabolic biomarker of neural stem/progenitor cells (NPCs) that allows the analysis of these cells in the live human brain. We have developed signal-processing algorithms that can detect metabolites present at very low concentration in the live human brain and can indicate possible pathways impaired in specific diseases. Herein, we present our strategy for both cellular and systems metabolomics, based on an integrative processing of the spectroscopy data that uses analytical tools from both metabolomic and spectroscopy fields. As an example of biomarker discovery using our approach, we present new data and discuss our previous findings on the NPC biomarker. Our studies link systems and cellular neuroscience through the functions of specific metabolites. Therefore, they provide a functional insight into the brain, which might eventually lead to discoveries of clinically useful biomarkers of the disease.
\end{abstract}

Early detection of disease is a pressing goal in clinical medicine. However, for most neurological disorders, specific biomarkers of the disease are not known. The diagnosis often relies on clinical or radiological criteria. The response to treatment is also qualitative. Therefore, during the past decade, the search for diagnostic biomarkers of specific disorders has escalated and has included genomics, proteomics, and, most recently, metabolomics analysis of a variety of disease states (Fiehn et al. 2001; German et al. 2005; Goodacre 2005; Hollywood et al. 2006).

Metabolomics, a nontargeted identification and quantification of a collection of metabolites in a biological system (the "metabolome"), is used to examine biochemical and metabolic processes in a given tissue or fluid (Fig. 1) (Fiehn 2002; Nicholson and Wilson 2003; Goodacre et al. 2004). The level of each metabolite within the metabolome depends on the specific physiological, developmental, and pathological state of a cell or tissue, and thus, the metabolome reflects tissue phenotype that results from both genetic and environmental influences (Gavaghan et al. 2000; Roessner et al. 2001; Phelps et al. 2002; Griffin 2004; Griffin and Nicholls 2006; Lindon and Holmes 2006). Therefore, by monitoring the biochemical status of an organism, one can indirectly assess its gene functions as well as the environmental effects on the genome expression (Fiehn et al. 2000; Raamsdonk et al. 2001; Harrigan and Goodacre 2003; Oksman-Caldentey and Saito 2005).
Such complex data collection has generally been accomplished using mass spectrometry or nuclear magnetic resonance (NMR) spectroscopy of body fluids or tissues in vitro, rather than of intact organs in a living organism (Beckonert et al. 2007). The complexity of the metabolome data sets has led to the development of different analytical strategies in order to discern its details, such as target analysis and metabolomic profiling (Goodacre 2004). Metabolomic fingerprinting, a subcategory of metabolomic profiling, represents a scan of a large number of intracellular metabolites with the aim of finding a specific signature of a certain tissue or certain state of the tissue. Metabolomic footprinting, on the other hand, identifies secreted metabolites in a given sample and is mostly used in microbial metabolomics (Kell et al. 2005).

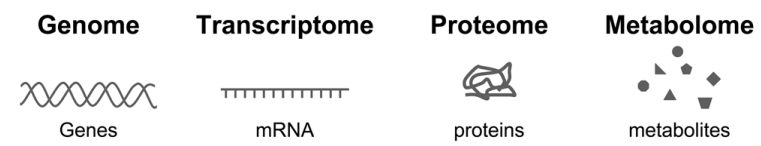

Figure 1. Flow chart of main compartments within the cell. Genomics investigates the genome pool of the cell, which consists of about 25,000 genes. mRNA $(\sim 100,000)$ is investigated through transcriptomics, whereas proteins $(\sim 1,000,000)$ are investigated through proteomics. Metabolomics investigates the metabolome, which consists of about 2500 small molecules, such as amino acids, peptides, lipids, and sugars. 
A correlation of unique metabolomic fingerprints with certain disease states not only indicates predictive biomarkers, but can also provide novel information related to the underlying disease biology. Although mostly investigated in cancer cell biology (Griffin and Shockcor 2004; Cheng and Pohl 2007), the feasibility of metabolomic profiling for assessment of a variety of conditions has been demonstrated in other diseases (Moolenaar et al. 2003; Prabakaran et al. 2004; Lindon and Holmes 2006; Holmes et al. 2008; Li et al. 2008). One of the most significant applications has been the investigation of the use of ${ }^{1} \mathrm{H}-\mathrm{NMR}$ spectra of human sera for rapid, accurate, and noninvasive assessment of the severity of coronary artery disease (Brindle et al. 2002). More recent studies have indicated the existence of human metabolic phenotypes related to diet and hypertension (Brindle et al. 2003; Rezzi et al. 2007; Holmes et al. 2008). In addition, the use of metabolomics has been explored in drug-safety monitoring and toxicology (DeGraaf 1998; Waters et al. 2001; Nicholson et al. 2002; Griffin and Bollard 2004; Mortishire-Smith et al. 2004; Coen et al. 2008). Therefore, metabolomics represents a noninvasive and medically applicable set of techniques for detection of low quantities of known metabolites and identification of unknown compounds, and it provides a great opportunity for biomarker discovery and the prospect of an increasingly important role in medicine and biotechnology.

\section{METABOLOMICS DATA ACQUISITION}

To obtain the metabolomic profile of a desired sample, complex data collection has generally been accomplished using mass spectrometry or NMR spectroscopy of body fluids or tissues in vitro in order to detect low quantities of known metabolites and identify unknown compounds (Beckonert et al. 2007; Lenz and Wilson 2007). The NMR-based approach is high-throughput and allows rapid data acquisition of large data sets. In addition, the preparation of the samples is minimal, which increases the highthroughput nature of this form of metabolomics data acquisition. The principle of NMR is based on the fact that nuclei with odd atomic or mass numbers act like magnets oriented toward or against an external magnetic field-a property known as nuclear spin (Hatada and Kitayama 2004). The ${ }^{1} \mathrm{H}-\mathrm{NMR}$ uses the external magnetic field to align the nuclear spins of the protons within the sample. To detect these spins, the nuclei are exposed to a rapidly varying electromagnetic field whereby, at one very specific frequency (denoted resonance), the spins absorb this electromagnetic radiation. After a pulse of electromagnetic radiation, the spins return to equilibrium at time constants T1 (longitudinal or spin-spin relaxation) and T2 (transversal or spin-lattice relaxation). During the nuclei relaxation process, there is an emission of radiation, and it is this emission that is detected as the ${ }^{1} \mathrm{H}-\mathrm{NMR}$ signal. The exact resonance frequency, however, will vary from one proton to the next, depending on the exact chemical structure of the molecule, such as the molecular bonds that bind the protons and the number of carbon atoms in the molecule and their spatial relationship to the protons (Ross and
Bluml 2001; Hatada and Kitayama 2004). Therefore, ${ }^{1} \mathrm{H}-$ NMR records a unique signal for each chemically distinct hydrogen nucleus. Recently, an improved ${ }^{1} \mathrm{H}-\mathrm{NMR}$ methodology has gained widespread use for (semi)heterogeneous samples, such as tissues. Magic angle spinning (MAS) NMR applies rotation along the magic angle of $54.7^{\circ}$ at high speed, and this significantly enhances sample homogeneity and spectral resolution (Garrod et al. 1999; Griffin et al. 2003). However, none of these techniques can be used for the analysis of metabolomic profiles of intact organs in a living organism. Therefore, despite its great value for metabolomic analysis of isolated samples and fluids, NMR spectroscopy cannot provide in vivo data that would be useful for metabolic profiling of intact organs in health and disease.

A correlate of ${ }^{1} \mathrm{H}-\mathrm{NMR}$ spectroscopy that provides information about the metabolic status of a tissue in vivo is proton magnetic resonance spectroscopy $\left({ }^{1} \mathrm{H}-\mathrm{MRS}\right)$, a noninvasive magnetic resonance imaging (MRI) modality that investigates the functional and dynamic status of the tissue in real time. Therefore, complements the anatomically based MRI and other MRI modalities that provide information about a variety of physiological parameters, such as blood flow, tissue perfusion, and water mobility. In addition, ${ }^{1} \mathrm{H}$-MRS surpasses conventional MRI imaging because it can detect metabolic abnormalities that might precede structural changes, therefore providing insights into the pattern of disease evolution and progression. Like NMR, MRS can also detect different nuclei, such as ${ }^{1} \mathrm{H},{ }^{31} \mathrm{P},{ }^{13} \mathrm{C},{ }^{15} \mathrm{~N},{ }^{19} \mathrm{~F}$, and ${ }^{23} \mathrm{Na}$, with ${ }^{1} \mathrm{H}$ and ${ }^{31} \mathrm{P}$ being the most frequently used. ${ }^{31} \mathrm{P}-\mathrm{MRS}$ was the first to be applied in in vivo brain spectroscopy and has been used to evaluate energy metabolism (Burt et al. 1979; Bernard et al. 1983). However, ${ }^{1} \mathrm{H}-\mathrm{MRS}$ has surpassed the use of ${ }^{31} \mathrm{P}$-MRS because it allows the detection of different biomarkers that are specific for neurons ( $N$-acetyl aspartate, NAA) and astrocytes (myoinositol, mI, and choline, Cho), the two most abundant cell types present in brain tissue (Ross and Bluml 2001). Thus, ${ }^{1} \mathrm{H}-\mathrm{MRS}$ has become the mainstay of human brain spectroscopy.

The first in vivo ${ }^{1} \mathrm{H}-\mathrm{MRS}$ of rat brain was acquired by Behar et al. (1983) and was followed by the first ${ }^{1} \mathrm{H}-\mathrm{MRS}$ of human brain by Bottomley et al. (1985). As shown in Figure 2, the ${ }^{1} \mathrm{H}-\mathrm{MRS}$ acquires data within a specific volume of the brain tissue, designated as volume or voxel of interest (VOI). Within the spectra, one can clearly discern metabolites that are present at high concentration in the brain tissue, such as NAA, Cho, $\mathrm{mI}$, and creatine (Cr), the energy metabolite that is most stable (Fig. 2). However, many more metabolites exist within the scanned VOI (Table 1), but, due to a variety of data acquisition and processing constraints, only several more can be reliably detected in human brain spectra, such as glutamate, GABA, and lactate. The main data acquisition constraints come from the overabundant presence of water in brain tissue and magnetic field inhomogeneity. The quality of ${ }^{1} \mathrm{H}-\mathrm{MRS}$ data greatly depends on the signal-to-noise ratio (SNR) because the method investigates not the water peak, but metabolites that are present in the brain tissue at much smaller concentrations. Therefore, historically, the first problem encountered in ${ }^{1} \mathrm{H}-\mathrm{MRS}$ was the presence of 

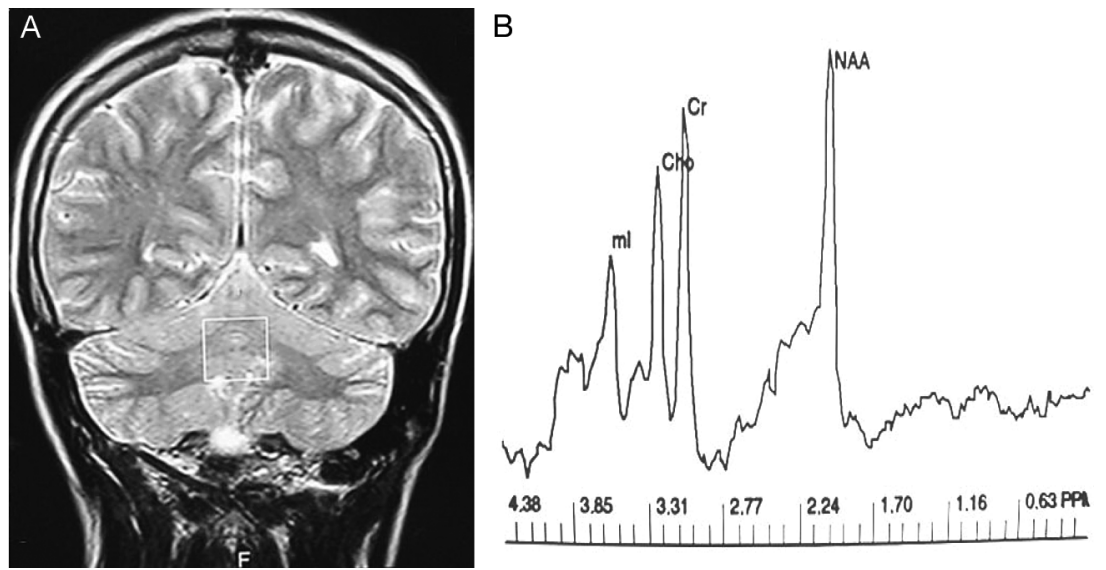

Figure 2. ${ }^{1} \mathrm{H}$-MRS acquisition of a single voxel spectroscopy. $(A)$ Coronal MRI scan with VOI in the cerebellum. Anatomical scan is done first to guide the positioning of the VOI within which the spectroscopy will be performed. $(B){ }^{1} \mathrm{H}-\mathrm{MRS}$ of the VOI in $A$, processed by FFT. Main metabolites are outlined: $N$-acetyl aspartate (NAA) is a biomarker of neurons, choline (Cho) and myoinositol (mI) are biomarkers of glial cells, and creatine (Cr) is a biomarker of energy metabolism. (VOI) Voxel of interest; (FFT) fast Fourier transform.

water, reaching up to $90 \mathrm{~mol} / \mathrm{liter}$ concentration and creating a large background signal that overshadowed the signals of all other metabolites present. To overcome this problem, a variety of techniques had to be developed that either did not excite the background water signal or suppressed it substantially (Bottomley et al. 1985). Furthermore, although NMR of intact tissue and tissue extracts has provided high separation of individual spectral peaks, the field inhomogeneity intrinsic to in vivo ${ }^{1} \mathrm{H}-\mathrm{MRS}$ causes broadening of the peaks and loss of significant detail about the individual metabolites. The analysis of such spectra would lead to unreliable results because metabolites of lower concentration may be hidden within the broad peaks and therefore pass undetected, and, if the peak area is used as a measure of metabolite concentration, broad peaks due to field inhomogeneity might lead

Table 1. Metabolites detected by spectroscopy in the live human and rodent brain as well as in tissue biopsies

\begin{tabular}{lc}
\hline Metabolite & Concentration range $\left(\mathrm{mmol} / \mathrm{kg}_{\mathrm{ww}}\right)$ \\
\hline Acetate & $0.4-0.8$ \\
NAA & $7.9-1.6$ \\
ATP & 3.0 \\
Alanine & $0.2-1.4$ \\
GABA & $1.3-1.9$ \\
Aspartate & $1.0-1.4$ \\
Choline (total) & $0.9-2.5$ \\
Creatine & $5.1-10.6$ \\
Glucose & 1.0 \\
Glutamate & $6.0-12.5$ \\
Glutamine & $3.0-5.8$ \\
Glycine & $0.4-1.0$ \\
Lactate & $0.3-0.6$ \\
Myoinositol & $3.8-8.1$ \\
Phosphocreatine & $3.2-5.5$ \\
Phosphorylcholine & 0.6 \\
Phosphorylethanolamine & $1.1-1.5$ \\
Pyruvate & 0.2 \\
\hline
\end{tabular}

\section{Adapted from Govindaraju et al. (2000)}

The list includes only the metabolites of $>0.5 \mathrm{mmol} / \mathrm{kg}_{\mathrm{ww}}$ concentration. to misleading values. To resolve this problem, a variety of techniques have been developed, from generating simulated echoes to localize different regions of the brain (Frahm et al. 1989; Keevil 2006) to improved shimming (i.e., optimization of the field inhomogeneity). Finally, to be detected by ${ }^{1} \mathrm{H}-\mathrm{MRS}$, a metabolite must be small $(<20$ $\mathrm{kDa}$ ), soluble, and mobile in the magnetic field (Ross and Bluml 2001). In an in vivo situation, a variety of conditions may change the solubility and/or mobility of a metabolite detected in vitro. Under such conditions, a metabolite visible by in vitro NMR might be invisible or erroneously increased by in vivo MRS. Fortunately, this problem has not been frequently encountered because major metabolites discovered by ${ }^{1} \mathrm{H}-\mathrm{NMR}$ in vitro, such as NAA, Cr, Cho, glutamate, $\mathrm{mI}$, and others, have all been detected by ${ }^{1} \mathrm{H}-\mathrm{MRS}$ in vivo in the human brain (Ross and Bluml 2001; Ross et al. 2006).

Despite the above pitfalls, ${ }^{1} \mathrm{H}-\mathrm{MRS}$, since its approval by the Federal Drug Administration (FDA) in 1993, has been used to identify and characterize metabolic changes associated with a variety of neurological diseases (Di Costanzo et al. 2007). The list is long and includes neurodegenerative diseases, cerebrovascular diseases, dementias, brain tumors, and demyelinating disorders, as well as rare diseases such as Canavan disease, Rasmunssen's encephalitis, creatine deficiency syndrome, and others (Bonavita et al. 1999; Lin et al. 2005). However, despite its value, ${ }^{1} \mathrm{H}$-MRS has not become a part of a routine clinical assessment because of the limited number of metabolites that can be analyzed using traditional signalprocessing methodologies. Fortunately, during the past few years, new analytical tools have emerged that allow for a more comprehensive analysis of ${ }^{1} \mathrm{H}-\mathrm{MRS}$ data. The new approaches come from the field of metabolomics as well as from new developments in the ${ }^{1} \mathrm{H}-\mathrm{MRS}$ field, where the search for more precise quantification methods applicable to data acquired by higher magnetic field strengths, such as 3T and 7T human MRI scanners, has been increasingly relevant. 


\section{METABOLOMICS SIGNAL PROCESSING}

Metabolomic signal processing consists of several steps: processing of raw data that allows for comparisons of different data sets, data mining that involves use of statistical tools for high-throughput analysis which identifies variables of interest, and quantitative measurements that provide both absolute and relative concentrations of selected metabolites which contribute mostly to the pathology investigated (Glassbrook et al. 2000; Dunn et al. 2005; Goodacre 2005). Herein, we describe some signal-processing algorithms used in metabolomics and ${ }^{1} \mathrm{H}-\mathrm{MRS}$.

The most widely used analytical methods for processing complex metabolomic data sets are based on multivariate statistical analysis. Principal component analysis (PCA) (Jolliffe 1986) is an unsupervised multivariate analysis that highlights the differences in a data set using a smaller number of factors, the principal components (PC) that capture the most variance in the data set. The first PC is the vector that accounts for the most variation in the data, and each observation is projected onto this line. The second PC is represented by another vector in $K$ dimensional space, which is orthogonal to the first PC, and represents the second largest variation in the data set. Many PCs may be obtained from a single data set, which consists of a multitude of related spectra; however, the variation quickly tapers off within a few PCs. Most often, the first ten PCs explain more than $90 \%$ of the variance. The PCA data are presented as a scores plot that reveals clustering in the observations based on similarities in the spectra. Thus, PCA provides a rapid means of visualizing and comparing data sets, where different clustering indicates the true variance among data classes. Additionally, a loadings plot is obtained from PCA that assists in the interpretation of the scores plot by highlighting the spectral regions responsible for clustering. In ${ }^{1} \mathrm{H}-\mathrm{MRS}$ data, it is expected that actual peaks, not noise, are the highest contributing factors (Goodacre et al. 2004).

Partial least squares - discriminant analysis (PLS-DA) is another analytical method widely used in multivariate analysis. Whereas PCA reduces the dimensionality of a multidimensional data set while retaining the characteristics of the data set that contribute most to its variance, PLS-DA is a regression extension of PCA that takes advantage of a priori class information to maximize the separation among groups of observations. Therefore, PLS-DA uses the basic principles of PCA but further minimizes the noise and maximizes the differences among classes. It is a compromise between the usual discriminant analysis and the discriminant analysis on the significant PC of the predictor variables. As such, PLS-DA has been used for predictive modeling of data sets and is especially suited to deal with a large number of predictors compared to the number of observations, which might pose a problem when analyzing complex metabolomic data sets (Perez-Enciso and Tenenhaus 2003). Although PCA and PLS-DA as well as other related multivariate analytical methods have been applied for analysis of the metabolomic data, they are not traditionally used for the analysis of ${ }^{1} \mathrm{H}-\mathrm{MRS}$ data.
To specifically process ${ }^{1} \mathrm{H}-\mathrm{MRS}$ data, one can use frequency- or time-domain-based methodologies. Herein, we outline the basic principles for ${ }^{1} \mathrm{H}$-MRS signal processing and the most frequently used software. First, the signals that carry information in ${ }^{1} \mathrm{H}-\mathrm{MRS}$ are exponentially decaying sinusoids, also known as free induction decay (FID) signals. They become smaller over time and are modeled as

$$
y_{n}=\sum_{k=1}^{K} a_{k} e^{\left(-d_{k}+j 2 \pi f_{k}\right) t}+w_{n}
$$

where $y_{n}$ are the measured signal samples; $n=0,1,2, \cdots$, $N-1 ; K$ is the number of decaying complex sinusoids in the model; $j=\sqrt{-1} ; a_{k}, d_{k}$, and $f_{k}$ are the amplitude, decay factor, and frequency of the $k$ th complex sinusoid, respectively; $t_{n}=n T_{s}$, where $T_{s}$ is the sampling interval; $w_{n}$ represents noise sample. In addition, more general models that account for imperfections of the signal acquisition process include a Gaussian decay factor and a combination of Lorentzian and Gaussian decays (Slotboom et al. 1998; Bartha et al. 1999).

In ${ }^{1} \mathrm{H}-\mathrm{NMR}$, a significant amount of prior knowledge exists about the parameters used in Equation 1, often derived by imposing constraints on these parameters. For example, the prior knowledge in ${ }^{1} \mathrm{H}-\mathrm{NMR}$ can be the information about frequencies and/or the decay factors of the metabolites, ratios of some of their amplitudes, or differences in their frequencies (DeGraaf 1998; Bartha et al. 1999). Obviously, with better prior knowledge, the methods for metabolite quantification yield more accurate results.

The most widely utilized method for quantification of ${ }^{1} \mathrm{H}-\mathrm{MRS}$ data is based on the peak frequency domain and integration of the areas under the peaks of Fourier-transformed data (Mierisova and Ala-Korpela 2001; Zandt et al. 2001). The fast Fourier transform (FFT) is an algorithm that converts the data in the time domain (i.e., the time constants of decay of the sinusoids) into the frequency domain, generating peaks identified on the basis of the frequency at which they resonate, known as the chemical shift. The chemical shift is a dimensionless value measured in parts per million (ppm) when compared with a reference resonance frequency, usually that of 3-trimethylsilylpropionic acid (TSP) or tetramethylsilane (TMS). The area under a peak is, in principle, proportional to the concentration of the metabolite that generates the peak (Meyer et al. 1988; DeGraaf 1998). However, methods based on peak area computation have low estimation accuracy and most do not use the model (Eq. 1) directly (Vanhamme et al. 1997). This is primarily due to problems that arise from the tails of metabolite spectra that overlap with the spectral peaks of interest. One method, however, that fits a linear combination of single metabolite spectra with an arbitrary line shape under the constraints of a common baseline has shown good results and is known as the linear combination of model (LCModel) (Provencher 2001). To incorporate maximum prior knowledge into the analysis, LCModel uses complete spectra rather than individual resonances. It is noninteractive and nearly model-free, and it accounts 
automatically for the baseline and provides both absolute and relative quantifications of the detected compounds. As such, it has gained wide acceptance for in vivo ${ }^{1} \mathrm{H}$ MRS processing (Jíru et al. 2003; Kanowski et al. 2004; Rosen and Lenkinski 2007).

Another group of methods for quantification of ${ }^{1} \mathrm{H}$ MRS data are known as time-domain methods (Vanhamme et al. 1999, 2001). Typically, these methods rely on model assumptions as in Equation 1. One of the first widely used time-domain interactive methods was the variable projection method (VARPRO; van der Veen et al. 1988). A method that improves the robustness and flexibility of the analysis is known as AMARES (Barkhuijsen et al. 1985; Vanhamme et al. 1997). Some of the improvements result from the use of prior knowledge and the possibility of fitting echo signals. An interactive software package that combines VARPRO and AMARES and allows for frequency selective filtering, correction of eddy currents artifacts, enhancement of FID signals, and linear prediction is known as the magnetic resonance user interface (MRUI) package. An important drawback of all of the interactive methods is their reliance on human assistance.

In addition to these interactive methods, there are black-box methods that are also based on time-domain processing. They use the linear prediction principle or state-space representations. From theory, it is known that the observations represented by Equation 1 can be approximately modeled by a high-order autoregressive (AR) model, i.e.,

$$
y_{n}=-\sum_{m=1}^{p} \alpha_{m} y_{n-m}+w_{n}
$$

where $p$ is the order of the AR model, $\alpha_{m}$ are its parameters, and $w_{n}$ is its driving noise. Because the information about the original parameters $a_{k}, d_{k}$, and $f_{k}$ is in the AR parameters, the objective is to estimate the $\alpha_{m}$ s and then deduce the values of $a_{k}, d_{k}$, and $f_{k}$. In principle, the $f_{k}$ s and $d_{k}$ s are first obtained from the $\alpha_{m}$ s and then substituted back in Equation 1, to allow for the estimation of $a_{k}$. A popular method for processing decayed sinusoids based on Equation 2 exploits the concept of singular-value decomposition (SVD) (Haacke et al. 1989). A straightforward application of SVD to ${ }^{1} \mathrm{H}-\mathrm{MRS}$ data first develops an overparametrized model such as that in Equation 2 and expresses it in a vector-matrix form, where the unknown vector is composed of the parameters $\alpha_{m}$. This vector multiplies data matrix whose elements are various samples of the data, $y_{n}$. After performing SVD of the data matrix, the rank of that matrix can be reduced by preserving only a subset of the largest singular values and equating the remaining ones with zero. With the rank-reduced matrix, a significant noise contribution is removed, better solutions for $\alpha_{m}$ are obtained, and, as a result, better estimates of $f_{k}$ and $d_{k}$ are produced. Finally, novel approaches for better estimation of metabolite quantitation are still being developed (Poullet et al. 2007) with the aim to optimize processing and fitting of the acquired spectra, as reviewed in a number of papers (Mierisova and AlaKorpela 2001; Provencher 2001; Vanhamme et al. 2001; Zandt et al. 2001).
Although the described methods are mostly used to quantify single-peak metabolites, a novel method has recently been developed for identifying metabolites with multiple peaks in the spectra (Cloarec et al. 2005). Statistical total correlation spectroscopy (STOCSY), the cross-correlation of correlation matrices, offers two major classes of correlations. One set is the resonance/peak pattern of individual components, which is charaterized by the highest correlation coefficient for any molecule, because all peak intensities vary together as the concentration of a particular molecule/metabolite varies across the data set. The added, important benefit of STOCSY is the next class of correlations that connects those components which vary positively and negatively in a concerted fashion. This is likely to happen if there are connections in the metabolic pathways or other reasons for coherences in pathophysiological processes reflected at the metabolic level. Therefore, STOCSY offers a unique analytical ability to provide information not only about the multipeak metabolites but also about metabolic pathways that might correlate during a certain cell/tissue state.

In summary, many signal-processing methodologies are now available for analysis of metabolomic data. Some of these processing methodologies are used for all "omics" data sets and some are used for quantification of single- and multipeak metabolites detected in the spectra. A combination of these methodologies provides comprehensive information not only about the patterns of differences among various data sets, but also about the most significant specific metabolites and specific pathways that correlate positively or negatively during the given state of the tissue. The outlined metabolomic methodologies have traditionally been applied to analyze NMRacquired data sets in vitro. However, they can also be applied to analyze ${ }^{1} \mathrm{H}-\mathrm{MRS}$ data sets in vivo, providing yet unexplored insights into the living brain metabolome.

\section{CELLULAR AND SYSTEM METABOLOMICS: A NOVEL APPROACH FOR BIOMARKER DISCOVERY}

Metabolomics studies have been limited by the almost exclusive systems biology approach and have not yet been able to translate systems data into concrete, clinically useful diagnostic and prognostic biomarkers of a disease. In contrast, we propose a more comprehensive use of metabolomics that aims to identify biomarkers through both cellular metabolomics (profiling of chosen cell types under in vitro conditions) and systems metabolomics (profiling of human tissue in vivo). Herein, we describe our strategy for identification of biomarkers of human brain disease(s), which combines the two methodologies. We also outline our signal-processing approach, which might provide qualitative and quantitative evaluation of individual metabolites and their contribution to the investigated condition in both healthy and diseased brain tissue.

Cellular metabolomics or the bottom-up approach (Fig. 3 ) is used to define both the whole metabolome and the unique metabolomic fingerprint of different cell types present in brain tissue in both normal and abnormal states. First, by using a $16 \mathrm{~T}(700 \mathrm{MHz}){ }^{1} \mathrm{H}-\mathrm{NMR}$, which provides 


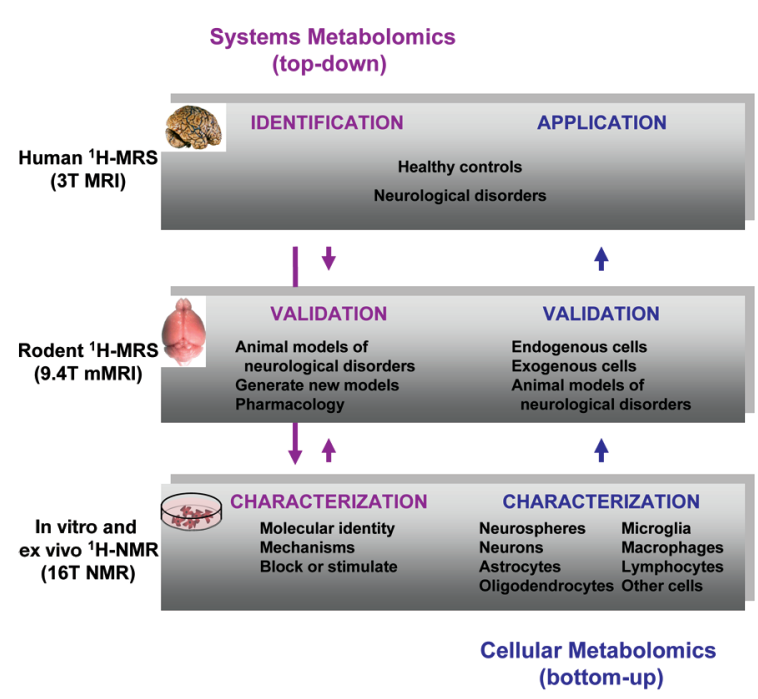

Figure 3. Flow chart of systems and cellular metabolomic strategies for biomarker discovery. A combination of such strategies readily translates clinically discovered biomarkers to cellular mechanistic and pharmacological studies, as well as in vitro gained knowledge to clinically applicable biomarkers. For example, systems metabolomics (top-down approach) uses ${ }^{1} \mathrm{H}$ MRS of the human brain to identify biomarkers of the disease. Once they are discovered, they can be investigated on the cellular level, through NMR of cells and tissues, and validated in animal models using ${ }^{1} \mathrm{H}$-MRS. On the other hand, cellular metabolomics (bottom-up approach) uses ${ }^{1} \mathrm{H}-\mathrm{NMR}$ to characterize cellular and molecular biomarkers in vitro. Once they are discovered, they can be validated in animal models through ${ }^{1} \mathrm{H}$-MRS spectroscopy and applied to human brain imaging for correlation with a specific disease.

the maximal spectral resolution, we can characterize very precisely the metabolomic profiles specific for different cell types. By analyzing the cellular metabolomic profiles, we can identify and characterize metabolites unique for each cell type in a healthy condition, thereby generating a database of cellular metabolomic fingerprints for any studied cell. To investigate whether a certain disease has an effect on the cellular metabolomic fingerprint, one can mimic a variety of disease conditions in culture and analyze both intracellular (fingerprint) and extracellular (footprint) changes of the metabolome. This is accomplished by the ${ }^{1} \mathrm{H}-\mathrm{NMR}$ of whole cells and cell extracts, as well as the extracellular conditioned media obtained during the course of the investigated condition. Furthermore, in this system, we can both investigate the mechanisms that lead to specific metabolomic dysfunction and test whether any $\operatorname{drug}(\mathrm{s})$ reverses the effects of the disease and recovers the normal cellular metabolome in vitro.

We can then directly translate our findings to in vivo ${ }^{1} \mathrm{H}$-MRS metabolomic profiling of the brain tissue. However, under the in vivo condition, relatively simple cellular and molecular metabolomic fingerprints are embedded in a complex tissue, where all cell types are present in a specific ratio. First, to test whether the cellular and molecular biomarkers retain their chemical shift in vivo, we can inject desired cells or molecules into the rodent cortex in vivo and then perform microMRI $\left({ }^{1} \mathrm{H}-\right.$ MRS) spectroscopy of the injected regions. This step is crucial for establishing the most optimal mMRI acquisition parameters for ${ }^{1} \mathrm{H}$-MRS. To validate the specificity of the obtained results, we use several well-established methodologies to confirm that signals obtained from the ${ }^{1} \mathrm{H}-\mathrm{MRS}$ spectroscopy indeed belong to the injected material. For example, we can perform ${ }^{1} \mathrm{H}-\mathrm{NMR}$ of the homogenized tissue obtained from the same animal that underwent mMRI, fluorescence-activated cell sorting (FACS) or enzyme-linked immunosorbent assay (ELISA) analysis of the cell types and molecules injected, and/or immunohistochemical staining of the cortical sections to correlate histological findings with the quantitative mMRI data. Therefore, animal models provide an invaluable tool for validation of metabolomic data translated from the in vitro studies and for their correlation with the changes at the genome and proteome levels that occur in the same animal model.

If expected metabolomic fingerprints are obtained repetitively and reliably in vivo, we can then investigate the biomarker dynamics in a disease state by using animal models of human disease with pathology known to involve the respective cells and molecules of interest. To validate these data, the same methodologies used for validation of exogenous biomarkers can be used. Moreover, in animal models, one can manipulate the disease in a variety of ways and then analyze the changes of individual biomarkers and the whole metabolome both qualitatively and quantitatively. Finally, both spacial and temporal studies can be done to estimate not only the degree of disease process and the presence of repair molecules and cells, but also their dynamic changes over time.

The most significant contribution of this strategy lies in its direct application to the imaging and characterization of the human brain metabolome. Through our integrative bottom-up approach, we have discovered a metabolite that is enriched in NPCs (Manganas et al. 2007). We have detected this metabolite in the live human brain, a significant breakthrough that might have direct impact on our ability to test the role of NPCs in a variety of neurological and psychiatric diseases. We can now investigate NPC density in any age group, from neonates to elderly, and correlate it with a variety of clinical, systemic, cognitive, radiological, and other measures in any disease of interest. Similarly, we can investigate other cellular and molecular biomarkers, once characterized and validated in our in vitro and in vivo studies (see Fig. 3), emphasizing a significant potential of this experimental approach.

Overall, our studies have demonstrated that cellular metabolomics can provide invaluable insights into the metabolome of isolated cells in vitro, which can be directly translated to the metabolomics of human brain tissue in vivo. However, cellular metabolomics might not offer sufficient insight into the complex changes that occur in diseased tissue in vivo, because those changes involve interactions of many cell types and molecules present within the tissue. Therefore, to investigate specific disease biomarkers in a most comprehensive way, in addition to cellular metabolomics, we need to use systems metabolomics (top-down approach) and to translate those findings to animal models and cellular levels (see Fig. 3). 
Using ${ }^{1} \mathrm{H}-\mathrm{MRS}$ of the human brain in vivo to acquire systems metabolomic data, we first investigate the patterns that differentiate the diseased tissue from the normal tissue. By dissecting the specific patterns down to individual metabolites, as outlined below, we can identify the most significant contributors to the metabolic differences observed, as well as correlate them within metabolic pathways that might be impaired in a given disease (Fig. 3). Once identified, specific patterns can be further investigated in animal models of a chosen disease using mMRI, and specific individual biomarkers or identified pathways can be investigated on a cellular level using NMR. As in the cellular metabolomic strategy, the in vitro systems are useful for characterization of the metabolites and pathways, identification of the mechanisms involved, and creation of pharmaceutical agents that affect those specific compounds. Investigations of animal models in vivo in the system metabolomic approach also aim to validate the data acquired in both human and cellular studies. Therefore, in our research, both cellular and system metabolomics converge in the animal models used to validate the in vitro and human in vivo data and to study biomarker and pathway identity and mechanisms that control them.

The key to our proposed strategy lies in signal processing that combines traditional metabolomic tools with algorithms used for ${ }^{1} \mathrm{H}-\mathrm{MRS}$ processing (Fig. 4). First, multivariate analysis, such as PCA, is used to process the complex data sets and to provide information about the principal components that contribute to the identified patterns of difference, whether these are detected in vitro or in vivo (Fig. 4). Such analysis of the metabolomic data is then faced with two key challenges: (1) It must determine whether the observed statistical patterns are meaningful, and (2) it must then translate these patterns into biologically relevant information in the form of metabolite identity and quantity (Weckwerth and Morgenthal 2005).

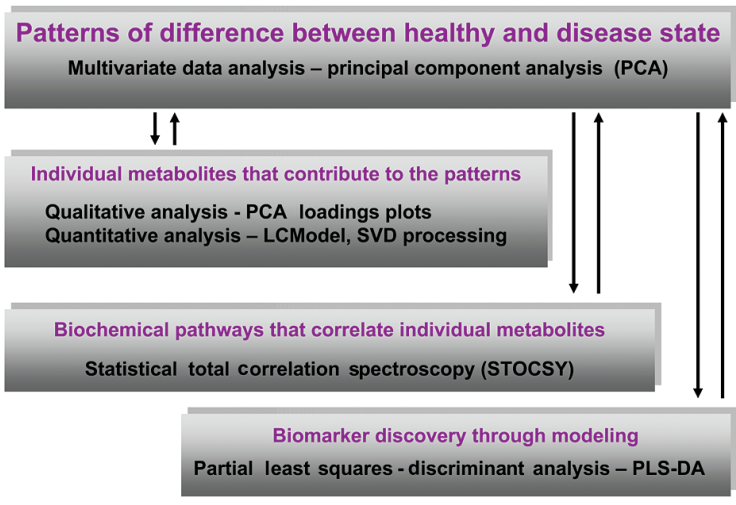

Figure 4. Flow chart of signal-processing tools used for metabolomic and spectroscopy signal processing. Such comprehensive analytical approach may lead to identification of patterns of difference between different samples as well as individual metabolites that contribute to the pattern difference and correlation between them. This analytical approach may ultimately identify and characterize specific diagnostic and prognostic biomarkers of a disease state. All of the data obtained may be used for predictive modeling. See main text for details.
PCA provides the loadings plots that may be used to identify individual metabolites that contribute mostly to the observed pattern difference. To investigate the specific metabolites discovered by the multivariate analysis and loadings plots, we apply already developed algorithms used in ${ }^{1} \mathrm{H}$-MRS processing.

Several signal-processing methods can be used to quantify individual metabolites with known frequency of oscillation, such as SVD and LCModel (Fig. 4). Our method of choice for quantitative analysis of metabolites with known frequency has been SVD-based, specifically designed to overcome the significant decline in biomarker concentration and spectroscopic resolution as we proceed from cells to live brain tissue. However, signal processing is very much limited by the quality of the data to be processed, particularly when the SNR is low. Spurious signals outside the region of interest can further contribute to the noise of the obtained spectra, and these effects may modify the final estimates of the cell and/or metabolite concentrations. Thus, to ensure reliable signal processing, many iterations within the selected frequency range must be performed to ascertain that signals are coming from the metabolite of interest. Another problem may arise if many cell types within the region of interest have the same biomarker and therefore contribute to the frequency band of interest. To minimize this confounder, the purity of the primary cultures must be ensured for best-quality data when investigating cellular biomarkers in vitro, and a combination of different cell types in vitro may be used to evaluate the anticipated in vivo conditions. Finally, a critical issue in data analysis is the probability of a false alarm, i.e., the probability of detecting biomarkers when there are none. Therefore, it is important to find the distribution of the estimation error from which we can deduce the sensitivity of the analytical method. These are all crucial performance specifications for any applied method of analysis, and they need to be precisely outlined to interpret the obtained results reliably.

Once we identify the specific cellular fingerprints through our in vitro analysis, we can use signal processing to isolate, amplify, and quantify single-cell signatures within complex metabolomic data of a chosen brain region. These signal-processing algorithms may enable the quantification of a variety of metabolites present in the tissue at low concentrations, such as those that indicate amino acid biochemistry; processing of proteins, sugars, and lipids; as well as the oxidoreductive status of the mitochondria. In addition, one can also apply correlative analysis such as custom-developed STOCSY to analyze metabolic pathways and metabolites that correlate in their expression pattern (up- or down-regulated) within the spectra. Such correlative analysis might provide useful information about the possible biochemical pathways that might be perturbed in the disease state. Finally, predictive modeling can be done using PLS-DA, which, in combination with all of the other analytical methodologies, might lead to the ultimate goal of our studies - a clinically useful application of specific metabolomic biomarker.

In summary, implementation of our experimental strategy and signal-processing methodologies might shed light on as yet undiscovered processes that may underline 
the particular brain disease one aims to investigate. Our approach may indicate specific metabolic differences between healthy and disease states or between different parts of the diseased brain. Furthermore, it might reveal specific metabolites and metabolic pathways that significantly contribute to the observed metabolic difference. Clinically, such studies might lead to the identification of diagnostic and prognostic biomarkers for individual symptoms or for the whole phenotype of the disease. Finally, specific metabolites and pathways may be further investigated on the cellular level, for both mechanistic and pharmacological studies, which could further lead to identification of the rescue mechanisms that might lead to the establishment of the normal metabolomic profiles both in vitro and in vivo.

\section{IMAGING OF NEURAL PROGENITOR CELLS: AN EXAMPLE OF METABOLOMICS BIOMARKER DISCOVERY}

Since their discovery in the human brain (Eriksson et al. 1998; Roy et al. 2000), NPCs have been envisioned as a new hope for a variety of neurological diseases. Even though their significant therapeutic capability has been widely recognized, the absence of well-defined markers that would distinguish them from other neural cell types has hampered their investigation in the live human brain. Until recently, methodologies for identification of NPCs in the living human brain have not been available, and without the ability to identify NPCs, the analysis of their fate and function in the human brain has been virtually impossible.

During the past few years, molecular and cellular MRI imaging has emerged as a new field for visualization of macromolecules and cells in living organisms and has been attempted for identification and tracking of NPCs (Bulte et al. 2002; Modo et al. 2005). Among several paramagnetic reagents, superparamagnetic iron oxide (SPIO) particles have been used in both molecular and cellular MRI studies. Several methodologies for conjugation of SPIO particles to transfection agents have been tested in order to enable efficient intracellular magnetic labeling (Arbab et al. 2003; Bulte and Kraitchman 2004; Bulte 2006). Migration of SPIO-labeled NPCs has been studied by mMRI in several animal models of human disease, such as stroke, glioma, and multiple sclerosis (Hoehn et al. 2002; Modo et al. 2004; Anderson et al. 2005; Ben-Hur et al. 2007). Recently, a clinical study has reported tracking of SPIO-labeled pancreatic islet grafts used to treat diabetic patients (Toso et al. 2008). Although no immediate side effects were observed, the authors recognized that iron overload might be the major obstacle for use of SPIO-based compounds (Toso et al. 2008). In addition to SPIO-based MRI imaging, positron emission tomography (PET) and single-photon emission computerized tomography (SPECT) scanning of ex vivo radiolabeled cells have been attempted and have led to a semiquantitative method for evaluation of cell trafficking (Adonai et al. 2002; Chin et al. 2003). However, all of the above techniques are applicable only to exogenous NPCs and do not provide the means for tracking endogenous NPCs. In addition, they cannot be used for imaging of infants and young children because radiolabeled reagents have not been approved by the FDA for children under 18 years of age. Therefore, there are currently no clinical highresolution imaging techniques that would enable spatiotemporal localization and tracking of unlabeled NPCs.

We have approached NPC imaging from the metabolomics standpoint and have applied a cellular metabolomics strategy to investigate metabolic biomarkers of NPCs and their progeny. First, ${ }^{1} \mathrm{H}-\mathrm{NMR}$ data were acquired for all individual cell types, grown as purified primary cultures in vitro (700-MHz Bruker NMR) (Manganas et al. 2007). As shown in Figure 5A, the raw spectra clearly indicate the differences in the metabolomes of NPCs and their progeny: neurons, astrocytes, and oligodendrocytes, the main cell types present in the brain. A major difference that can be deduced from the raw spectra is the presence of the $1.28 \mathrm{ppm}$ metabolite in the NPC spectra and not in the spectra of other cell types. To investigate the metabolomic pattern of each cell type, we performed multivariate analysis (SIMCA-P, Umetrics). The PCA confirmed a clear difference in metabolomic patterns among the four cell types (Fig. 5). Interestingly, each cell type clustered separately, indicating that their metabolic profiles were specific. Whereas astrocytes and oligodendrocytes separated from neurons along the principal component 2 (PC2), NPCs clustered away from all of them along the PC1 (Fig. 5B). One of the main metabolites contributing to the separation of NPCs from other cell types was the $1.28 \mathrm{ppm}$ metabolite, as seen in the PC1 loadings plot (Fig. 5D). Therefore, metabolomic profiling of NPCs indicates that they are clearly metabolically different from other neural cell types.

In addition to gaining the information on the NPC metabolomic profile, the first PCA indicated that clusters of astrocytes and oligodendrocytes are close together, suggesting that these cells share common metabolic compounds as compared to neurons and NPCs (Fig. 5B). However, the metabolic profiles of these cells have some differences, as indicated by further PCA processing. As shown in Figure 5C, astrocytes and oligodendrocytes cluster away from each other based on PC3. The metabolite that seems to contribute the most to this separation is choline, resonating at $3.23 \mathrm{ppm}$ and seen in the PC3 loadings plot (Fig. 5F).

We have further characterized the 1.28-ppm metabolite in terms of its quantification, specificity, and basic molecular identity (Manganas et al. 2007). To exemplify our strategy described earlier in the text, we show an example of our translation from in vitro NMR data to animal and human brain imaging of NPCs.

Translation of the in vitro data to in vivo brain tissue imaging faces significant challenges, such as considerable decline in magnet strength (from 16T Bruker NMR scanner to 9.4T mMRI Bruker scanner to 3T Phillips MRI scanner) associated with decreased SNR; spectroscopy of tissue as compared to spectroscopy of isolated cell types; and presence of our cells of interest, NPCs, in a limited concentration within a certain brain region (hippocampus) that presents its own intrinsic challenges for ${ }^{1} \mathrm{H}$-MRS acquisition (field inhomogeneity, close proximity to the cerebrospinal fluid, etc.). The data acquisition is essential for reliable processing because all signal-processing algorithms heavily depend on the SNR. Because an NPC 

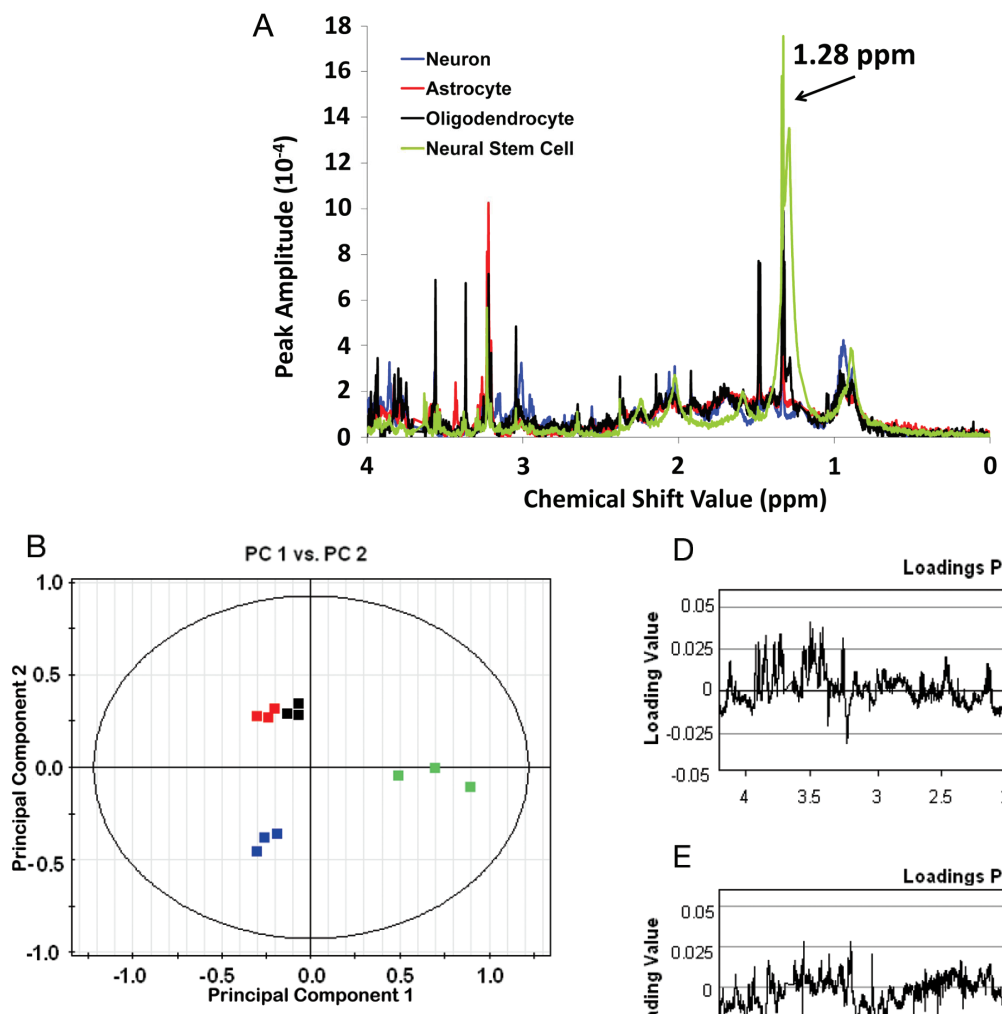

D
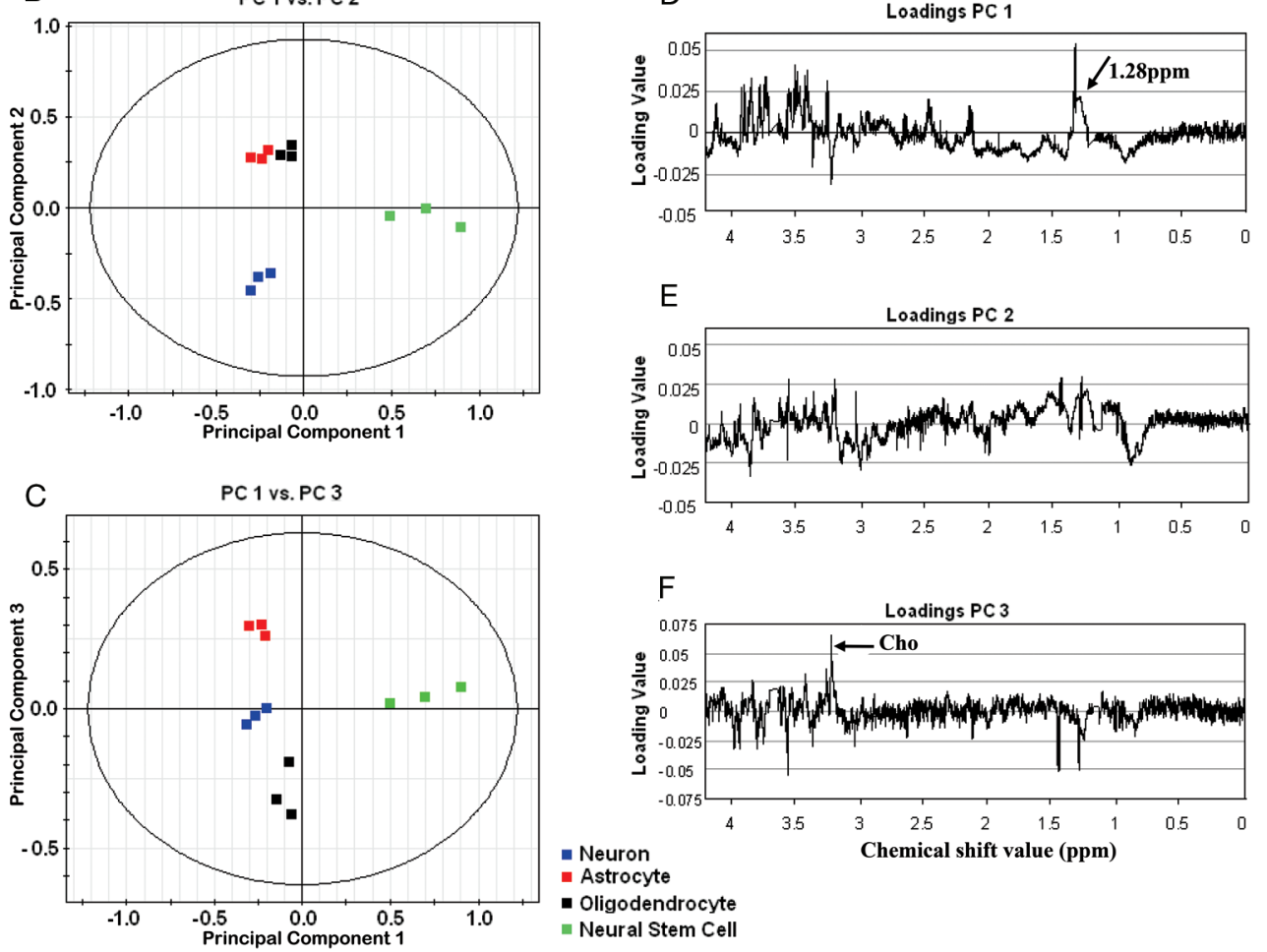

Neuron

- Oligodendrocyte

- Neural Stem Cell

Figure 5. Multivariate analysis of the neural cell types in vitro. (A) NMR spectra of individual cell types indicate differences among them, as well as abundant presence of the $1.28 \mathrm{ppm}$ metabolite that distinguishes NPCs from the other cell types. $(B)$ PCA of the NMR spectra of neural cell types indicates tight clustering of individual cell types as well as clear separation of different cell types. Specifically, NPCs separate from others along PC1, whereas astrocytes and oligodendrocytes separate from neurons along PC2. (C) PCA of the NMR spectra of neural cell types identifies principal component 3 as the main contributor for the metabolic difference between astrocytes and oligodendrocytes. $(D, E, F)$ Loadings plots for PC1, -2 , and -3. Loadings plots for each PC indicate variations of individual metabolites in the spectra and can be used to identify those metabolites that mostly contribute to the observed pattern difference. Loadings plot for the PC1 $(D)$ indicates that the $1.28 \mathrm{ppm}$ metabolite is one of the major contributors for the separation of the NPCs from the other cell types. Loadings plot for the PC3 $(F)$ indicates that choline (Cho) is one of the major contributors for the separation of the astrocytes from the oligodendrocytes.

metabolite, resonating at $1.28 \mathrm{ppm}$ is embedded within the noise when in vivo spectra are processed by the FFT, we have exploited a signal-processing algorithm based on SVD that enabled us to detect NPCs in vivo using high-resolution ${ }^{1} \mathrm{H}-\mathrm{MRS}$ of the rodent brain and the human brain (Manganas et al. 2007; Djurić et al. 2008).

Herein, we show two examples of detection of NPCs in vivo in an adult rat brain (Fig. 6) and in an adult human brain (Fig. 7). Meticulous placement of the VOI is crucial for proper ${ }^{1} \mathrm{H}-\mathrm{MRS}$ acquisition. For the mMRI of the rodent brain, the hippocampus (where NPCs reside) is close to the center of the magnetic field, and therefore shimming and ${ }^{1} \mathrm{H}-\mathrm{MRS}$ acquisition are "easier" than shimming and acquisition of the spectra in the cortex, which serves as a negative control (Fig. 6). The opposite is true for the human brain ${ }^{1} \mathrm{H}-\mathrm{MRS}$ - the hippocampal region is notoriously difficult for data acquisition, and this might lead to poor SNR and unreliable analysis. The examples of 

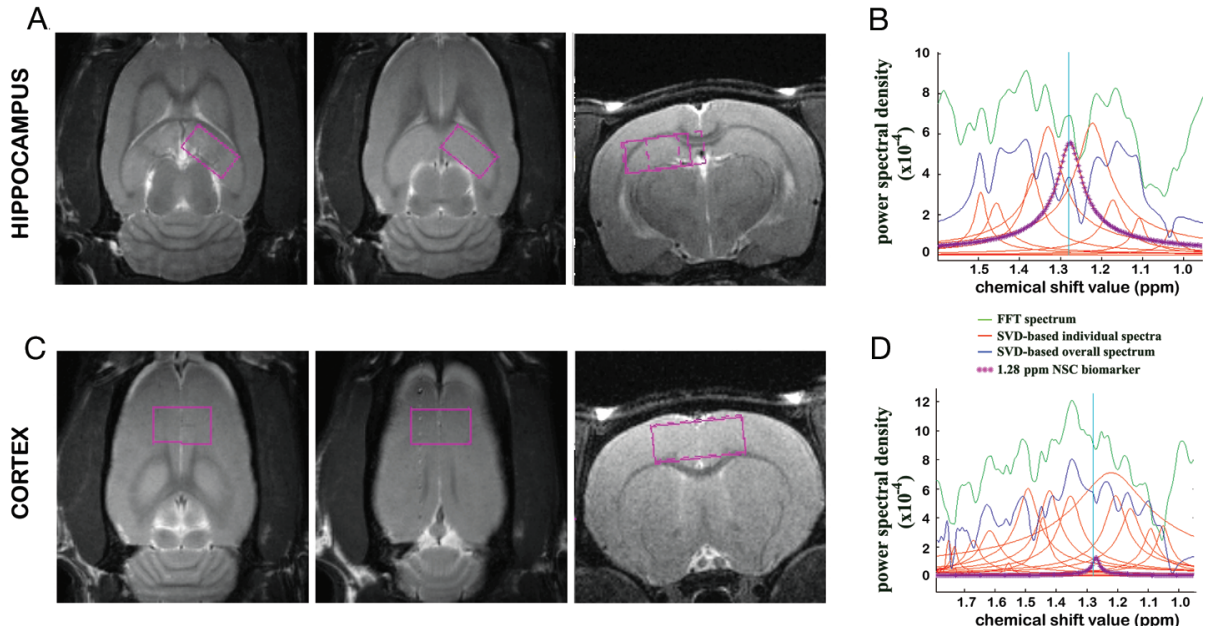

Figure 6. ${ }^{1} \mathrm{H}-\mathrm{MRS}$ of the rodent brain in vivo. (A) Coronal and axial images of the rat brain with localization of the VOI in the hippocampus. (B) Singular-value decomposition (SVD)-based signal processing of the spectra obtained in $A$. Modeling of the individual peaks outlines the $1.28 \mathrm{ppm}$ metabolite and enables its quantification. (C) Coronal and axial images of the rat brain with localization of the VOI in the cortex. $(D)$ SVD-based signal processing of the spectra obtained in $C$. Modeling of the individual peaks within the similar range as in $B$ indicates minimal presence of the $1.28 \mathrm{ppm}$ metabolite in the cortex.

the SVD analysis of both rodent and human spectra are shown in Figures 6 and 7, respectively. Processing is done as described by Manganas et al. (2007). Briefly, the data are imported into MatLab and water is removed. In addition, signals whose tails might compromise the detection of the 1.28-ppm metabolite are also removed. We then search for the 1.28-ppm signal within a specific range $(1.28 \pm 0.02$ ppm) and quantify it based on its amplitude. The processing is slightly different for rodent and human data because they differ in acquisition parameters and model order (Manganas et al. 2007). Both rodent and human in vivo data can be scrutinized by other analytical methods of multivariate analyses, which may yield more information about pattern differences among the different brain regions.
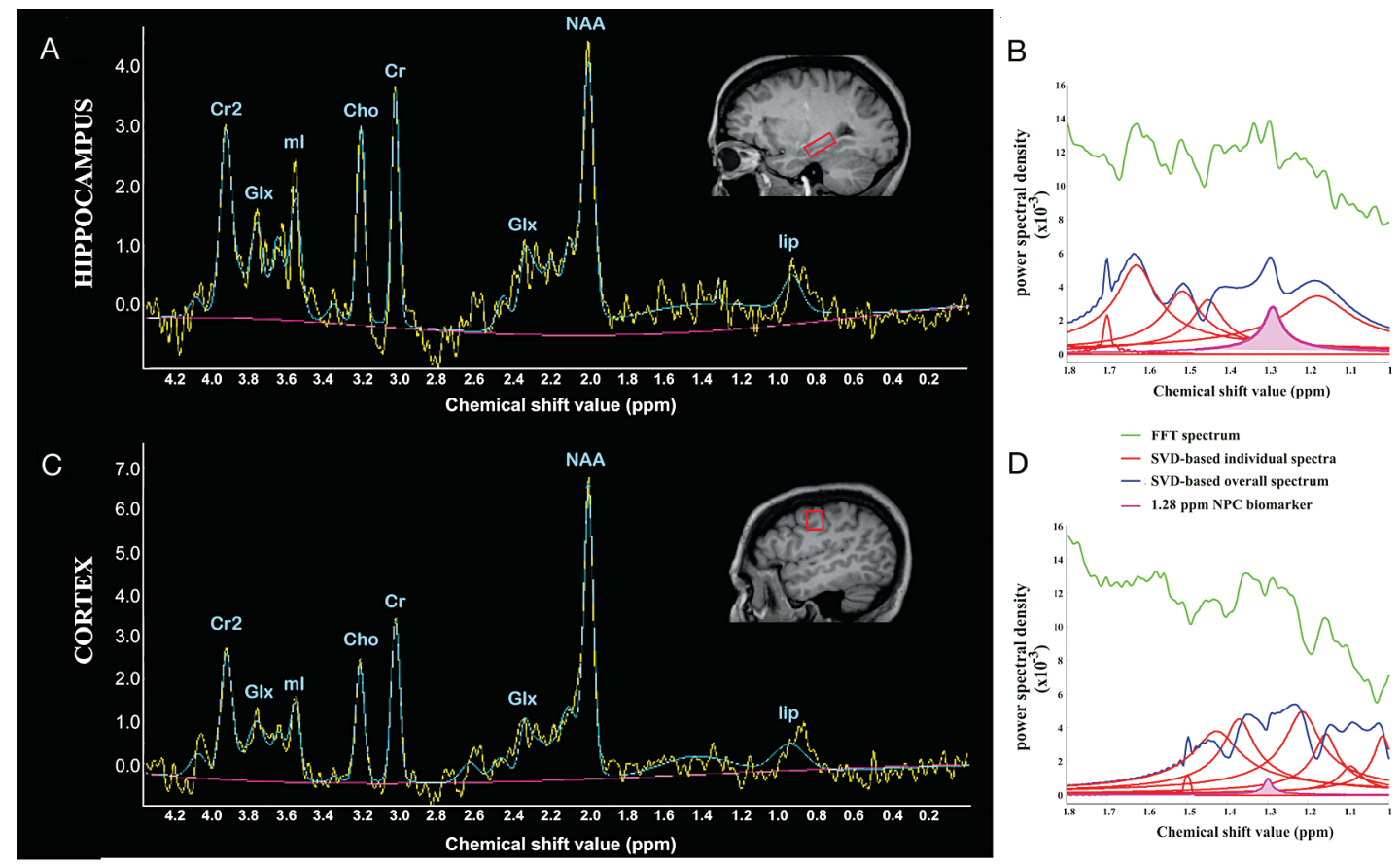

Figure 7. ${ }^{1} \mathrm{H}-\mathrm{MRS}$ of the human brain in vivo. $(A)$ Sagittal MRI scan of the human brain with localization of the VOI in the hippocampus. FFT spectra are shown with major metabolites indicated $(\mathrm{Glx}=$ glutamate and glutamine). $(B)$ Singular-value decomposition (SVD)-based signal processing of the spectra obtained in $A$. Modeling of the individual peaks outlines the 1.28 ppm metabolite and enables its quantification. (C) Sagittal MRI scan of the human brain with localization of the VOI in the cortex. FFT spectra are shown. $(D)$ SVD-based signal processing of the spectra obtained in $C$. Modeling of the individual peaks within the same range as in $B$ indicates minimal presence of the $1.28 \mathrm{ppm}$ metabolite in the cortex. 


\section{CONCLUSION}

One of the biggest challenges in biomedical science is the translation of basic scientific accomplishments to clinical practice. Although drug or device developments have established principles of transition from in vitro to animal in vivo to human applications, other fields of medicine have not yet provided the means of clear transference. Our outlined strategy for experimental design and signal processing using metabolomics and spectroscopy tools might offer such means and a direct translation of biomarkers characterized in vitro to clinical applications in vivo. Similarly, our approach can provide an unprecedented insight into the functional status of live human brain tissue and can translate this information to more readily available validation models, such as animal models and studies at the cellular level. Furthermore, although a well-known correlate of high-resolution NMR, ${ }^{1} \mathrm{H}-\mathrm{MRS}$ spectroscopy has never been scrutinized using complex data analysis paradigms well established for ${ }^{1} \mathrm{H}-\mathrm{NMR}$ metabolomics. Application of metabolomics analytical methods to ${ }^{1} \mathrm{H}$-MRS in conjunction with other spectroscopy signal-processing tools might therefore lead to a potential wealth of functional human brain ${ }^{1} \mathrm{H}-\mathrm{MRS}$ tissue-profiling data. We hope that our introduction of the concept of cross-fertilization among different disciplines, such as metabolomics and ${ }^{1} \mathrm{H}-\mathrm{MRS}$, in terms of signal processing and data correlation, will prompt further research in these areas, which ultimately may lead to new discoveries that will improve our understanding of human brain diseases.

\section{ACKNOWLEDGMENTS}

This work was supported by NINDS R21NS05875-1 and 5K08 NS044276, and U.S. Army Medical Research grant DAMD170110754 (M.M.S.); T32DK07521-16 (L.N.M.); NINDS R01-NS32764, NARSAD, Seraph Foundation, Hartman Foundation, Hope for Depression Foundation, and Hazan Foundation (G.E.); Department of Energy FWP MO-065 (H.B.); and National Science Foundation CCF-0515246 and Office of Naval Research N00014-06-1-0012 (P.D.).

\section{REFERENCES}

Adonai, N., Nguyen, K.N., Walsh, J., Iyer, M., Toyokuni, T., Phelps, M.E., McCarthy, T., McCarthy, D.W., and Gambhir, S.S. 2002. Ex vivo cell labeling with 64Cu-pyruvaldehydebis(N4-methylthiosemicarbazone) for imaging cell trafficking in mice with positron-emission tomography. Proc. Natl. Acad. Sci. 99: 3030-3035.

Anderson, S.A., Glod, J., Arbab, A.S., Noel, M., Ashari, P., Fine, H.A., and Frank, J.A. 2005. Noninvasive MR imaging of magnetically labeled stem cells to directly identify neovasculature in a glioma model. Blood 105: 420-425.

Arbab, A.S., Bashaw, L.A., Miller, B.R., Jordan, E.K., Lewis, B.K., Kalish, H., and Frank, J.A. 2003. Characterization of biophysical and metabolic properties of cells labeled with superparamagnetic iron oxide nanoparticles and transfection agent for cellular MR imaging. Radiology 229: 838-846.

Barkhuijsen, H., de Beer, R., Bovee, W.M., Creyghton, J.H., and van Ormondt, D. 1985. Application of linear prediction and singular value decomposition (LPSVD) to determine NMR frequencies and intensities from the FID. Magn. Reson. Med. 2: $86-89$.
Bartha, R., Drost, D.J., and Williamson, P.C. 1999. Factors affecting the quantification of short echo in-vivo $1 \mathrm{H} \mathrm{MR}$ spectra: Prior knowledge, peak elimination, and filtering. NMR Biomed. 12: 205-216.

Beckonert, O., Keun, H.C., Ebbels, T.M., Bundy, J., Holmes, E. Lindon, J.C., and Nicholson, J.K. 2007. Metabolic profiling, metabolomic and metabonomic procedures for NMR spectroscopy of urine, plasma, serum and tissue extracts. Nat. Protoc. 2: 2692-2703.

Behar, K.L., den Hollander, J.A., Stromski, M.E., Ogino, T., Shulman, R.G., Petroff, O.A., and Prichard, J.W. 1983. Highresolution $1 \mathrm{H}$ nuclear magnetic resonance study of cerebral hypoxia in vivo. Proc. Natl. Acad. Sci. 80: 4945- 4948.

Ben-Hur, T., van Heeswijk, R.B., Einstein, O., Aharonowiz, M., Xue, R., Frost, E.E., Mori, S., Reubinoff, B.E., and Bulte, J.W. 2007. Serial in vivo MR tracking of magnetically labeled neural spheres transplanted in chronic EAE mice. Magn. Reson. Med. 57: 164-171.

Bernard, M., Canioni, P., and Cozzone, P.J. 1983. Study of in vivo cellular metabolism by phosphorus 31 nuclear magnetic resonance (transl.). Biochimie 65: 449-470.

Bonavita, S., Di Salle, F., and Tedeschi, G. 1999. Proton MRS in neurological disorders. Eur. J. Radiol. 30: 125-131.

Bottomley, P.A., Edelstein, W.A., Foster, T.H., and Adams, W.A. 1985. In vivo solvent-suppressed localized hydrogen nuclear magnetic resonance spectroscopy: A window to metabolism? Proc. Natl. Acad. Sci. 82: 2148-2152.

Brindle, J.T., Antti, H., Holmes, E., Tranter, G., Nicholson, J.K., Bethell, H.W., Clarke, S., Schofield, P.M., McKilligin, E., Mosedale, D.E., and Grainger, D.J. 2002. Rapid and noninvasive diagnosis of the presence and severity of coronary heart disease using 1H-NMR-based metabonomics. Nat. Med. 8: 1439-1444.

Brindle, J.T., Nicholson, J.K., Schofield, P.M., Grainger, D.J., and Holmes, E. 2003. Application of chemometrics to $1 \mathrm{H}$ NMR spectroscopic data to investigate a relationship between human serum metabolic profiles and hypertension. Analyst 128: $32-36$.

Bulte, J.W. 2006. Intracellular endosomal magnetic labeling of cells. Methods Mol. Med. 124: 419-439.

Bulte, J.W. and Kraitchman, D.L. 2004. Iron oxide MR contrast agents for molecular and cellular imaging. NMR Biomed. 17: 484-499.

Bulte, J.W., Duncan, I.D., and Frank, J.A. 2002. In vivo magnetic resonance tracking of magnetically labeled cells after transplantation. J. Cereb. Blood Flow. Metab. 22: 899-907.

Burt, C.T., Cohen, S.M., and Barany, M. 1979. Analysis with intact tissue with 31P NMR. Annu. Rev. Biophys. Bioeng. 8: $1-25$.

Cheng, L.L. and Pohl, U. 2007. The role of NMR-based metabolomics in cancer. In Handbook of metabonomics and metabolomics (ed. J.C. Lindon et al.), pp. 345-374. Elsevier, Amsterdam.

Chin, B.B., Nakamoto, Y., Bulte, J.W., Pittenger, M.F., Wahl, R., and Kraitchman, D.L. 2003. In oxine labelled mesenchymal stem cell SPECT after intravenous administration in myocardial infarction. Nucl. Med. Commun. 24: 1149-1154.

Cloarec, O., Dumas, M.E., Craig, A., Barton, R.H., Trygg, J., Hudson, J., Blancher, C., Gauguier, D., Lindon, J.C., Holmes, E., and Nicholson, J. 2005. Statistical total correlation spectroscopy: An exploratory approach for latent biomarker identification from metabolic 1H NMR data sets. Anal. Chem. 77: 1282-1289.

Coen, M., Holmes, E., Lindon, J.C., and Nicholson, J.K. 2008. NMR-based metabolic profiling and metabonomic approaches to problems in molecular toxicology. Chem. Res. Toxicol. 21: 9-27.

DeGraaf, R.A. 1998. In vivo NMR spectroscopy: Principles and techniques. Wiley, New York.

Di Costanzo, A., Trojsi, F., Tosetti, M., Schirmer, T., Lechner, S.M., Popolizio, T., and Scarabino, T. 2007. Proton MR spectroscopy of the brain at 3 T: An update. Eur. Radiol. 17: 1651-1662.

Djurić, P.M., Benveniste, H., Wagshul, M.E., Henn, F., Enikolopov, G., and Maletić-Savatić, M. 2008. Response to 
comments on "Magnetic resonance spectroscopy identifies neural progenitor cells in the live human brain." Science 321: 640.

Dunn, W.B., Bailey, N.J., and Johnson, H.E. 2005. Measuring the metabolome: Current analytical technologies. Analyst 130: 606-625.

Eriksson, P.S., Perfilieva, E., Bjork-Eriksson, T., Alborn, A.M., Nordborg, C., Peterson, D.A., and Gage, F.H. 1998. Neurogenesis in the adult human hippocampus. Nat. Med. 4: 1313-1317.

Fiehn, O. 2002. Metabolomics: The link between genotypes and phenotypes. Plant Mol. Biol. 48: 155-171.

Fiehn, O., Kopka, J., Dormann, P., Altmann, T., Trethewey, R.N., and Willmitzer, L. 2000. Metabolite profiling for plant functional genomics. Nat. Biotechnol. 18: 1157-1161.

Fiehn, O., Kloska, S., and Altmann, T. 2001. Integrated studies on plant biology using multiparallel techniques. Curr. Opin. Biotechnol. 12: 82-86.

Frahm, J., Bruhn, H., Gyngell, M.L., Merboldt, K.D., Hanicke, W., and Sauter, R. 1989. Localized high-resolution proton NMR spectroscopy using stimulated echoes: Initial applications to human brain in vivo. Magn. Reson. Med. 9: 79-93.

Garrod, S., Humpfer, E., Spraul, M., Connor, S.C., Polley, S., Connelly, J., Lindon, J.C., Nicholson, J.K., and Holmes, E. 1999. High-resolution magic angle spinning 1H NMR spectroscopic studies on intact rat renal cortex and medulla. Magn. Reson. Med. 41: 1108-1118.

Gavaghan, C.L., Holmes, E., Lenz, E., Wilson, I.D., and Nicholson, J.K. 2000. An NMR-based metabonomic approach to investigate the biochemical consequences of genetic strain differences: Application to the C57BL10J and Alpk:ApfCD mouse. FEBS Lett. 484: 169-174.

German, J.B., Hammock, B.D., and Watkins, S.M. 2005. Metabolomics: Building on a century of biochemistry to guide human health. Metabolomics 1: 3-9.

Glassbrook, N., Beecher, C., and Ryals, J. 2000. Metabolic profiling on the right path. Nat. Biotechnol. 18: 1142-1143.

Goodacre, R. 2004. Metabolic profiling: Pathways in discovery. Drug Discov. Today 9: 260-261.

Goodacre, R. 2005. Metabolomics shows the way to new discoveries. Genome Biol. 6: 354.

Goodacre, R., Vaidyanathan, S., Dunn, W.B., Harrigan, G.G., and Kell, D.B. 2004. Metabolomics by numbers: Acquiring and understanding global metabolite data. Trends Biotechnol. 22: $245-252$

Govindaraju, V., Young, K., and Maudsley, A.A. 2000. Proton NMR chemical shifts and coupling constants for brain metabolites. NMR Biomed. 13: 129-153.

Griffin, J.L. 2004. Metabolic profiles to define the genome: Can we hear the phenotypes? Philos. Trans. R. Soc. Lond. B Biol. Sci. 359: 857-871.

Griffin, J.L. and Bollard, M.E. 2004. Metabonomics: Its potential as a tool in toxicology for safety assessment and data integration. Curr. Drug Metab. 5: 389-398.

Griffin, J.L. and Nicholls, A.W. 2006. Metabolomics as a functional genomic tool for understanding lipid dysfunction in diabetes, obesity and related disorders. Pharmacogenomics 7: 1095-1107.

Griffin, J.L. and Shockcor, J.P. 2004. Metabolic profiles of cancer cells. Nat. Rev. Cancer 4: 551-561.

Griffin, J.L., Pole, J.C., Nicholson, J.K., and Carmichael, P.L. 2003. Cellular environment of metabolites and a metabonomic study of tamoxifen in endometrial cells using gradient high resolution magic angle spinning 1H NMR spectroscopy. Biochim. Biophys. Acta 1619: 151-158.

Haacke, E.M., Liang, Z.P., and Izen, S.H. 1989. Constrained reconstruction: A superresolution, optimal signal-to-noise alternative to the Fourier transform in magnetic resonance imaging. Med. Phys. 16: 388-397.

Harrigan, G.G. and Goodacre, R., eds. 2003. Metabolic profiling: Its role in biomarker discovery and gene function analysis. Kluwer, Boston.

Hatada, K. and Kitayama, T., eds. 2004. Basic principles of NMR. In NMR spectroscopy of polymers, pp. 1-34. Springer, New York
Hoehn, M., Kustermann, E., Blunk, J., Wiedermann, D., Trapp, T., Wecker, S., Focking, M., Arnold, H., Hescheler, J., Fleischmann, B.K., Schwindt, W., and Buhrle, C. 2002. Monitoring of implanted stem cell migration in vivo: A highly resolved in vivo magnetic resonance imaging investigation of experimental stroke in rat. Proc. Natl. Acad. Sci. 99: $16267-16272$.

Hollywood, K., Brison, D.R., and Goodacre, R. 2006. Metabolomics: Current technologies and future trends. Proteomics 6: 4716-4723.

Holmes, E., Loo, R.L., Stamler, J., Bictash, M., Yap, I.K., Chan, Q., Ebbels, T., De Iorio, M., Brown, I.J., Veselkov, K.A., et al. 2008. Human metabolic phenotype diversity and its association with diet and blood pressure. Nature 453: 396-400.

Jírů, F., Dezortová, M., Burian, M., and Hájek, M. 2003. The role of relaxation time corrections for the evaluation of long and short echo time 1H MR spectra of the hippocampus by NUMARIS and LCModel techniques. Magma 16: 135-143.

Jolliffe, I.T. 1986. Principal component analysis. SpringerVerlag, New York.

Kanowski, M., Kaufmann, J., Braun, J., Bernarding, J., and Tempelmann, C. 2004. Quantitation of simulated short echo time $1 \mathrm{H}$ human brain spectra by LCModel and AMARES. Magn. Reson. Med. 51: 904-912.

Keevil, S.F. 2006. Spatial localization in nuclear magnetic resonance spectroscopy. Phys. Med. Biol. 51: R579-R636.

Kell, D.B., Brown, M., Davey, H.M., Dunn, W.B., Spasic, I., and Oliver, S.G. 2005. Metabolic footprinting and systems biology: The medium is the message. Nat. Rev. Microbiol. 3: 557-565.

Lenz, E.M. and Wilson, I.D. 2007. Analytical strategies in metabonomics. J. Proteome Res. 6: 443-458.

Li, M., Wang, B., Zhang, M., Rantalainen, M., Wang, S., Zhou, H., Zhang, Y., Shen, J., Pang, X., Wei, H., et al. 2008. Symbiotic gut microbes modulate human metabolic phenotypes. Proc. Natl. Acad. Sci. 105: 2117-2122.

Lin, A., Ross, B.D., Harris, K., and Wong, W. 2005. Efficacy of proton magnetic resonance spectroscopy in neurological diagnosis and neurotherapeutic decision making. NeuroRx 2: $197-214$.

Lindon, J.C. and Holmes, E. 2006. A survey of metabonomics approaches for disease characterization. In Handbook of metabonomics and metabolomics (ed. J.C. Lindon et al.), pp. 413-442. Elsevier, Amsterdam.

Manganas, L.N., Zhang, X., Li, Y., Hazel, R.D., Smith, S.D., Wagshul, M.E., Henn, F., Benveniste, H., Djurić, P.M., Enikolopov, G., and Maletić-Savatić, M. 2007. Magnetic resonance spectroscopy identifies neural progenitor cells in the live human brain. Science 318: 980-985.

Meyer, R.A., Fisher, M.J., Nelson, S.J., and Brown, T.R. 1988. Evaluation of manual methods for integration of in vivo phosphorus NMR spectra. NMR Biomed. 1: 131-135.

Mierisova, S. and Ala-Korpela, M. 2001. MR spectroscopy quantitation: A review of frequency domain methods. NMR Biomed. 14: 247-259.

Modo, M., Mellodew, K., Cash, D., Fraser, S.E., Meade, T.J., Price, J., and Williams, S.C. 2004. Mapping transplanted stem cell migration after a stroke: A serial, in vivo magnetic resonance imaging study. Neuroimage 21: 311-317.

Modo, M., Hoehn, M., and Bulte, J.W. 2005. Cellular MR imaging. Mol. Imaging 4: 143-164.

Moolenaar, S.H., Engelke, U.F., and Wevers, R.A. 2003. Proton nuclear magnetic resonance spectroscopy of body fluids in the field of inborn errors of metabolism. Ann. Clin. Biochem. 40: $16-24$.

Mortishire-Smith, R.J., Skiles, G.L., Lawrence, J.W., Spence, S., Nicholls, A.W., Johnson, B.A., and Nicholson, J.K. 2004. Use of metabonomics to identify impaired fatty acid metabolism as the mechanism of a drug-induced toxicity. Chem. Res. Toxicol. 17: 165-173.

Nicholson, J.K. and Wilson, I.D. 2003. Opinion: Understanding "global" systems biology: Metabonomics and the continuum of metabolism. Nat. Rev. Drug. Discov. 2: 668-676.

Nicholson, J.K., Connelly, J., Lindon, J.C., and Holmes, E. 2002. Metabonomics: A platform for studying drug toxicity 


\section{METABOLOMICS OF NEURAL PROGENITOR CELLS}

and gene function. Nat. Rev. Drug Discov. 1: 153-161.

Oksman-Caldentey, K.M. and Saito, K. 2005. Integrating genomics and metabolomics for engineering plant metabolic pathways. Curr. Opin. Biotechnol. 16: 174-179.

Perez-Enciso, M. and Tenenhaus, M. 2003. Prediction of clinical outcome with microarray data: A partial least squares discriminant analysis (PLS-DA) approach. Hum. Genet. 112: 581-592.

Phelps, T.J., Palumbo, A.V., and Beliaev, A.S. 2002. Metabolomics and microarrays for improved understanding of phenotypic characteristics controlled by both genomics and environmental constraints. Curr. Opin. Biotechnol. 13: 20-24.

Poullet, J.B., Sima, D.M., Simonetti, A.W., De Neuter, B., Vanhamme, L., Lemmerling, P., and Van Huffel, S. 2007. An automated quantitation of short echo time MRS spectra in an open source software environment: AQSES. NMR Biomed. 20: $493-504$.

Prabakaran, S., Swatton, J.E., Ryan, M.M., Huffaker, S.J., Huang, J.T., Griffin, J.L., Wayland, M., Freeman, T., Dudbridge, F., Lilley, K.S., et al. 2004. Mitochondrial dysfunction in schizophrenia: Evidence for compromised brain metabolism and oxidative stress. Mol. Psychiatry 9: 684-697, 643.

Provencher, S.W. 2001. Automatic quantitation of localized in vivo 1H spectra with LCModel. NMR Biomed. 14: 260-264.

Raamsdonk, L.M., Teusink, B., Broadhurst, D., Zhang, N., Hayes, A., Walsh, M.C., Berden, J.A., Brindle, K.M., Kell, D.B., Rowland, J.J., et al. 2001. A functional genomics strategy that uses metabolome data to reveal the phenotype of silent mutations. Nat. Biotechnol. 19: 45-50.

Rezzi, S., Ramadan, Z., Martin, F.P., Fay, L.B., van Bladeren, P., Lindon, J.C., Nicholson, J.K., and Kochhar, S. 2007. Human metabolic phenotypes link directly to specific dietary preferences in healthy individuals. J. Proteome Res. 6: 4469-4477.

Roessner, U., Luedemann, A., Brust, D., Fiehn, O., Linke, T., Willmitzer, L., and Fernie, A. 2001. Metabolic profiling allows comprehensive phenotyping of genetically or environmentally modified plant systems. Plant Cell 13: 11-29.

Rosen, Y. and Lenkinski, R.E. 2007. Recent advances in magnetic resonance neurospectroscopy. Neurotherapeutics 4: $330-345$.

Ross, B. and Bluml, S. 2001. Magnetic resonance spectroscopy of the human brain. Anat. Rec. 265: 54-84.
Ross, B.D., Coletti, P., and Lin, A. 2006. Magnetic resonance spectroscopy of the brain: Neurospectroscopy. In Clinical magnetic resonance imaging (ed. R.R. Edelman et al.), pp. 1840-1901. Saunders, Philadelphia.

Roy, N.S., Wang, S., Jiang, L., Kang, J., Benraiss, A., HarrisonRestelli, C., Fraser, R.A., Couldwell, W.T., Kawaguchi, A., Okano, H., Nedergaard, M., and Goldman, S.A. 2000. In vitro neurogenesis by progenitor cells isolated from the adult human hippocampus. Nat. Med. 6: 271-277.

Slotboom, J., Boesch, C., and Kreis, R. 1998. Versatile frequency domain fitting using time domain models and prior knowledge. Magn. Reson. Med. 39: 899-911.

Toso, C., Vallee, J.P., Morel, P., Ris, F., Demuylder-Mischler, S., Lepetit-Coiffe, M., Marangon, N., Saudek, F., James Shapiro, A.M., Bosco, D., and Berney, T. 2008. Clinical magnetic resonance imaging of pancreatic islet grafts after iron nanoparticle labeling. Am. J. Transplant. 8: 701-706.

van der Veen, J.W., de Beer, R., Luyten, P.R., and van Ormondt, D. 1988. Accurate quantification of in vivo 31P NMR signals using the variable projection method and prior knowledge. Magn. Reson. Med. 6: 92-98.

Vanhamme, L., van den Boogaart, A., and Van Huffel, S. 1997. Improved method for accurate and efficient quantification of MRS data with use of prior knowledge. J. Magn. Reson. 129: $35-43$.

Vanhamme, L., Van Huffel, S., Van Hecke, P., and van Ormondt, D. 1999. Time-domain quantification of series of biomedical magnetic resonance spectroscopy signals. $J$. Magn. Reson. 140: 120-130.

Vanhamme, L., Sundin, T., Hecke, P.V., and Huffel, S.V. 2001. MR spectroscopy quantitation: A review of time-domain methods. NMR Biomed. 14: 233-246.

Waters, N.J., Holmes, E., Williams, A., Waterfield, C.J., Farrant, R.D., and Nicholson, J.K. 2001. NMR and pattern recognition studies on the time-related metabolic effects of $\alpha$-naphthylisothiocyanate on liver, urine, and plasma in the rat: An integrative metabonomic approach. Chem. Res. Toxicol. 14: 1401-1412.

Weckwerth, W. and Morgenthal, K. 2005. Metabolomics: From pattern recognition to biological interpretation. Drug Discov. Today 10: 1551-1558.

Zandt, H., van Der Graaf, M., and Heerschap, A. 2001. Common processing of in vivo MR spectra. NMR Biomed. 14: 224-232. 


\section{$\overbrace{\mathrm{CSH}}^{\infty} \mathrm{C}$ Cold Spring Harbor Symposia SYMPOSIA}

\section{Metabolomics of Neural Progenitor Cells: A Novel Approach to Biomarker Discovery}

M. Maletic-Savatic, L.K. Vingara, L.N. Manganas, et al.

Cold Spring Harb Symp Quant Biol 2008 73: 389-401 originally published online November 6, 2008 Access the most recent version at doi:10.1101/sqb.2008.73.021

References This article cites 82 articles, 9 of which can be accessed free at: http://symposium.cshlp.org/content/73/389.full.html\#ref-list-1

\section{License}

Email Alerting Receive free email alerts when new articles cite this article - sign up in the Service box at the top right corner of the article or click here. 